\title{
Researching Teams: Nothing's Going to Change Our World
}

\author{
Arwen H. Decostanza ${ }^{1 *}$ \\ Gia A. Dirosa ${ }^{1}$ \\ Sean E. Rogers ${ }^{2}$ \\ Andrew J. Slaughter ${ }^{1}$ \\ Armando X. Estrada ${ }^{1}$
}

${ }^{1}$ U.S. Army Research Institute for the Behavioral and Social Sciences

${ }^{2}$ Rutgers University

*correspondence: arwen.h.decostanza@us.army.mil

U.S. Army Research Institute (DAPE-ARI-FS)

6010 Frankford Street, Office 221A

Aberdeen Proving Ground, MD 21005

As researchers focused on enhancing the performance of complex teams within military organizations, we offer a uniquely applied perspective to the issues raised in the focal article (Tannenbaum, Mathieu, Salas, \& Cohen, 2012). We concur with Tannenbaum and colleagues that a gap between research and practice exists. To bridge this gap, we propose that we need to evaluate how variables affecting team effectiveness manifest, evolve, and affect performance in complex teams utilizing existing theory within and outside of our discipline. Moreover, we propose that more sophisticated methodological approaches that result in improvements in our ability to measure the phenomena of interest are necessary. Before elaborating on our proposed ideas, we pause to consider whether anything has changed and if anything is missing. 


\section{Has Anything Changed?}

We agree with Tannenbaum et al. (2012) that the environment in which teams operate has changed. However, we contend that many characteristics and conditions highlighted in the focal article are not new or different. Consider the example of the Persian Gulf incident of 1988 involving the U.S.S. Vincennes, where a civilian airliner was misidentified and shot down, killing all passengers and crew. This incident involved teams of teams working together over time in complex, fluid, dynamic, and distributed environments, where individuals and teams were responsible for decision making (McIntyre \& Salas, 1995). Recent military operations in both Afghanistan and Iraq have these same characteristics and conditions. For example, a provincial reconstruction team (PRT) may integrate combat personnel with civilian government personnel and contractors to perform a mission to restore services to a village or city area. Not unlike the teams on the U.S.S. Vincennes, PRTs must coordinate with diverse and changing teams of individuals to operate in complex contexts characterized by high operational tempo and dynamic deployment cycles that may require modularization of individuals and units. Thus, based on our work with military teams, we argue that many of these characteristics and conditions have been present all along, but their salience is what has changed. Accordingly, we question whether changes in the salience of these characteristics and conditions warrant reconsideration of our constructs, theories, and research.

\section{Is Anything Missing?}

Tannenbaum et al. describe complexities of teams well. However, an additional characteristic emerges in our research on military teams that is important to advance the study on teams. Multiplex interdependence describes the nature of the interdependencies that exist both 
among members of a team and across teams within a multiteam environment. Rather than a standard model of interdependence, which can be characterized as pooled, sequential, reciprocal, or intensive, interdependence among military teams evolves over time in a multidimensional (e.g., resource, task, goal, and interpersonal), multilevel (within and between teams at same and different hierarchical levels), and multiorganizational (spanning across organizations) fashion. For example, a PRT may need to coordinate with Officers and staff within the military, coordinate with individuals from both government and nongovernment organizations, incorporate guidance and resources from the civil and military chain of command, and coordinate up and down chains of command within attached units to accomplish their missions. Moreover, the nature of this interdependence may evolve as tasks progress, as team members gain experience, and as external factors change the nature of the operational environment. Thus, multiplex interdependence adds a layer of complexity that is critical to understand how teams must function in an increasingly complex operational environment. Accordingly, we propose that team researchers must begin to examine the complex, temporally evolving nature of interdependence to advance our understanding of the complexity surrounding modern teams.

\section{How Can We Bridge the Gap Between Research and Practice?}

Tannenbaum et al. highlight a number of important areas in which our knowledge remains limited. We contend that to enhance knowledge and bridge the gap between research and practice we must (a) utilize existing theory on team effectiveness to study complex teams,

(b) capitalize on theories from other related domains to better understand team performance, and

(c) advance our methodological approaches to the study of teams within complex environments.

\section{Utilizing Existing Theory on Team Effectiveness}


Much of the existing lab-based research has relied on what Tannenbaum et al. refer to as "traditional" teams (i.e., 3- to 4-person teams with stable membership, performing less complex tasks). This state of affairs may have less to do with the awareness of the complexities of realworld teams and more to do with sample size restrictions, available research methods, and the current state of the literature. Real-world teams may be more complex, but they are still teams at a fundamental level. Thus, existing theories of team effectiveness should be used as the building blocks for new research.

Literature on teams has reached consensus on a number of constructs that affect team effectiveness, including variables such as composition (e.g., size and member competencies), leadership, emergent states (e.g., cohesion, trust, and cognition), and processes (e.g., monitoring, back-up behavior, coordination, and conflict; Marks, Mathieu, \& Zaccaro, 2001; Salas, Sims, \& Burke, 2005). Instead of developing new theories of complex teams, bridging the gap between research and practice requires us to think critically about how these constructs manifest, evolve, and affect performance within complex interdependent systems. We contend that defining the team in terms of the multiplex interdependencies necessary to accomplish the task provides a structure for exploring the impact of additional complexities on these constructs.

\section{Capitalizing on Theories in Related Domains}

The themes identified by Tannenbaum et al. highlight the need to create more complex structures to describe teams. These complex structures require us to expand our thinking beyond "small group theories" and consider applicable theories in related domains to account for more complex forms of teams (e.g., multiteam systems, organizations as collectives of teams, and teams as networked organizations). 
Complex teams possess many structural, operational, and environmental attributes that make them as much "organization-like" as they are "team-like," suggesting the need for more applications of organizational theories to the team context. Hollenbeck et al. (2002) applied structural contingency theory to teams, suggesting that team structure should match the external organizational environment. Similarly, leveraging research on the impact of culture (organizational or national) on organizational performance could provide a lens for understanding factors that influence performance in complex teams such as competing organizational goals, cultural diversity, and procedural compatibility (Connaughton \& Shuffler, 2007). By utilizing what we already know about organizations and understanding its relevancy within complex teams, we can expand on existing theories.

Beyond organizational theory, other research domains could provide valuable building blocks for new research on complex teams. Strategic human resource management research may provide insights into design and implementation practices to motivate and encourage adaptability within complex teams. Network science can help us understand how interactions within and between teams predict critical team outcomes related to cognition, affect, and behavior. Complex adaptive systems approaches may help us define, measure, and model critical states and stochastic processes in complex teams. This list is not exhaustive but is meant to stimulate our thinking about the variety of existing theoretical perspectives applicable to today's teams.

\section{Advancing Measurement Methodologies}

A critical element for bridging the gap between research and practice involves advancing our measurement methodologies. To study complex teams characterized by multiplex interdependence, changing composition, ill-defined boundaries, diverse technologies, and 
nontraditional leadership and governance structures, the set of measurement techniques currently used in the majority of team research needs to be expanded.

From a measurement and modeling perspective, researchers of complex teams are faced with fundamental challenges whose solutions rely on understanding the inherent multiplex interdependencies of the team. In complex teams, rather than defining team membership as an a priori fixed categorical factor, researchers may need to find ways to estimate the membership of teams (which may overlap, coalesce, or diverge across time) based purely on observable patterns of social interactions and task dependencies. In such situations, individuals' memberships in various latent "teams" will not be categorical, static, and deterministic, but range across a (possibly dynamic and probabilistic) continuum. In addition, for teams without easily identifiable boundaries, or defined by complex, evolving dependency structures at multiple levels (e.g., social, cognitive, and task), more traditional ways of defining group-level constructs are difficult to apply both conceptually and analytically. Researchers must make use of new statistical and conceptual approaches that allow them to identify and differentiate basic patterns of intra- and interteam dependencies in relevant social relationships, work tasks, and knowledge structures; model dynamic changes in these dependencies at multiple levels of analysis simultaneously; and link key aspects of these models to traditional team constructs.

To some extent, the required conceptual and statistical frameworks for such models already exist and are currently being used in team research, albeit in limited fashion. Network analysis uses the mathematics of graph theory to measure and analyze patterns of edges (e.g., interactions or dependencies) that link vertices (e.g., different types of elements or nodes in the network). In traditional teams, simple types of networks (e.g., social networks) and topological 
features (e.g., centrality) are the focus. However, complex teams require much more complicated types of networks that include multiplex networks (multiple types of ties between nodes), multimode networks (multiple categories of nodes), cognitive social structures (cognitive networks embedded in social networks), and dynamic networks (changing ties and composition).

\section{Practicing What We Preach}

Within our applied research, we rely on existing literature to provide a foundation for defining and examining critical constructs and incorporate diverse theoretical perspectives (e.g., organizational theories applied to cohesion and adaptive performance in complex teams and network and multiteam systems theories to understand emergent dynamics) to help us take into account the complexity of military teams. We are developing unobtrusive measures of teamrelated constructs and relying on advanced statistical methods such as exponential random graph modeling (including models for dynamic and multi-relational networks) and epidemiological models and methods to further our conceptual and methodological approaches. These are some of the ways we are attempting to bridge the gap between research and practice. We have a long road ahead, but remain optimistic that existing theories and research will help us pave the way to new insights. 


\section{Authors Note:}

The views expressed in this paper are those of the authors and do not necessarily reflect the official policy or position of the Department of the Army, the Department of Defense, the U.S. Government, or any of its agencies. 


\section{References}

Connaughton, S. L., \& Shuffler, M. L. (2007). Multinational/ multicultural distributed teams: A review and future research agenda. Small Group Research, 38, 387-412.

Hollenbeck, J. R., Moon, H., Ellis, A. P. J., West, B. J., Ilgen, D. R., Sheppard, L., Wagner, J. A., III. (2002). Structural contingency theory and individual differences: Examination of external and internal person-team fit. Journal of Applied Psychology, 87, 599-606.

Marks, M. A., Mathieu, J. E., \& Zaccaro, S. J. (2001). A temporally based framework and taxonomy of team processes. Academy of Management Review, 26, 356-376.

McIntyre, R. M., \& Salas, E. (1995). Measuring and managing for team performance: Emerging principles from complex environments. In R. A. Guzzo \& E. Salas (Eds.), Team effectiveness and decision making in organizations (pp. 9-45). San Francisco, CA: Jossey Bass.

Salas, E., Sims, D. E., \& Burke, C. S. (2005). Is there a big five in teamwork? Small Group Research, 36, 555-599.

Tannenbaum, S. I., Mathieu, J. E., Salas, E., \& Cohen, D. (2012). Teams are changing: Are research and practice evolving fast enough? Industrial and Organizational Psychology: Perspectives on Science and Practice, 5, 2-24. 